\title{
METODOLOGÍA DE APLICACIÓN DE CMMI SERVICIOS EN PEQUEÑAS Y MEDIANAS EMPRESAS
}

\section{IMPLEMENTATION METHODOLOGY OF CMMI FOR SERVICES IN SMALL AND MEDIUM SIZED ORGANIZATIONS}

Mariana Palacios López ${ }^{1}$

Victor Gisbert Soler ${ }^{2}$

1. Graduada en Ingeniería Industrial (Escuela de Ingeniería de Antioquia - Colombia). Master Universitario en Ingeniería de Organización y Logística (Universidad Politécnica de Valencia - (España). E-mail: mapalo3@epsa.upv.es

2. Doctor Ingeniero Industrial. Departamento de Estadística e Investigación Operativa Aplicadas y Calidad. Universidad Politécnica de Valencia. (España). E-mail: vgisber@eio.upv.es

Citación sugerida:

Palacios López, M. y Gisbert Soler, V. (2017). Metodología de aplicación de CMMI servicios en pequeñas y medianas empresas. 3C Empresa: investigación y pensamiento crítico, Edición Especial, 92-100. DOI: <http://dx.doi.org/10.17993/3cemp.2017.especial.92-100/>. 


\section{RESUMEN}

En el presente artículo se propone una metodología de aplicación de CMMI Servicios en PYMES (Pequeñas y Medianas Empresas), comenzando con la búsqueda y comparación de antecedentes, para posteriormente explicar los conceptos básicos del modelo y plantear una metodología con base a contribuciones de otros autores, incluyendo aportaciones propias. Finalmente, se realiza un análisis de las ventajas de aplicación en PYMES, para llegar a unas conclusiones generales.

\section{ABSTRACT}

This article proposes an implementation methodology of CMMI for Services in SME (Small and Medium Size Enterprises), starting with the check up and comparison of the background, subsequently explain the basic concepts of the model and propose a methodology based on contributions of different authors, including own contributions. Finally, make a review of the application advantages in SME, in order to reach general conclusions.

\section{PALABRAS CLAVE}

CMMI, Servicios, Modelo, Mejora de procesos.

\section{KEY WORDS}

CMMI, Services, Model, Process Improvement. 


\section{INTRODUCCIÓN}

CMMI (Capability Maturity Model Integration) se trata de la integración de modelos de madurez de capacidades, el cual consta de diferentes ramas y campos de aplicación, entre los cuales se encuentran: CMMI para el Desarrollo (CMMI-DEV o CMMI for Development), CMMI para la adquisición (CMMI-ACQ o CMMI for Acquisition) y CMMI para los Servicios (CMMI-SVC o CMMI for Services).

En el presente artículo se elabora un análisis de la rama CMMI Servicios, el cual es un modelo de mejora y evaluación de procesos, basado en la aplicación de buenas prácticas de $\mathrm{CMMI}$, diseñado para proporcionar una guía en las actividades que requieren gestionar, establecer y entregar servicios de calidad a los clientes y usuarios finales.

Debido a que las pequeñas y medianas empresas se encuentran en un entorno altamente exigente, donde la competencia crece constantemente, se hace necesaria la aplicación de modelos, en este caso CMMI-SVC, que ayuden a evaluar y mejorar sus procesos, de tal manera que ofrezcan servicios de calidad a sus clientes y permita que crezcan de manera equilibrada y sostenida, asegurando y consolidando la mejora en el tiempo.

\section{ANTECEDENTES}

Respecto al tema tratado se han encontrado diferentes referencias y antecedentes, los cuales se describen y se analizan a continuación, para tomarlos como base para el planteamiento de la metodología de aplicación de CMMI Servicios en PYMES.

- Antecedente 1: Reporte técnico de CMMI Servicios, Versión 1.3.

Se caracteriza por proporcionar metas y prácticas genéricas en las diferentes áreas de proceso, iniciando con una explicación de la constelación $\mathrm{CMMI}$, pasando por la descripción de las áreas de proceso tratados en la metodología, así como la integración de todas ellas en un modelo completo, explicando los niveles de madurez y capacidad que se pueden alcanzar en la entrega de los servicios. Adicionalmente, expone la relación entre las diferentes áreas de proceso y describe las vías de adopción y uso de CMMI-SVC para la mejora de los procesos.

- Antecedente 2: Uso del área de proceso Service Delivery (SD) de CMMI for Services, versión 1.3 como guía para servicios en bibliotecas universitarias en el Valle del Cauca. En este proyecto de grado se aborda con gran detalle el área de proceso mencionada, proponiendo un modelo de implementación a partir de CMMI, comenzando por los requisitos previos y documentación requerida por parte de la biblioteca, adaptando el área de entrega de servicio al campo de aplicación de su tesis, para finalizar con la guía metodológica que se debería usar para analizar el estado de los servicios, elaborar propuestas, aprobar y crear planes de implementación. Este antecedente coincide con el anterior en que utiliza la teoría expuesta respecto a una de las áreas de proceso del reporte técnico, desglosándola y aplicándola al tipo de organización estudiada. 


\section{- Antecedente 3: Modelo de verificación y validación basado en CMMI.}

Contiene una introducción al modelo en sus niveles 2 y 3 para posteriormente plantear una metodología para verificar y validar el software basado en dichos estándares. Este antecedente coincide con el primero en cuanto a que aplica la base del reporte técnico para mejorar la calidad en la industria del software. Sin embargo, como no es aplicado a la prestación de un servicio sino al desarrollo de software, las áreas de proceso abordadas en el artículo son diferentes a las explicadas en el antecedente 1, es decir, se basa en las áreas del CMMI-DEV más no del CMMI-SVC.

- Antecedente 4: Estudio exploratorio de porqué las organizaciones no adoptan CMMI. Este estudio expresa que las organizaciones no aplican CMMI por diversas razones, entre las cuales se encuentran: la empresa es pequeña, los servicios son costosos, no tienen tiempo o se encuentran utilizando otro enfoque de mejora de procesos de software (SPI por sus siglas en inglés). Concluyen que las compañías, en general, no adoptan el modelo porque es inviable, más no porque no sea benéfico para las mismas. Este antecedente se relaciona con los anteriores en cuanto a que realiza un análisis de la aplicación real de los modelos $\mathrm{CMMI}$, considerando que el interés por implantarlo en una empresa, depende en gran medida de su tamaño.

\section{METODOLOGÍA}

Iniciemos este apartado por entender a grandes rasgos las características del modelo CMMI Servicios, para finalizar con la propuesta de una metodología para implementarlo en pequeñas y medianas empresas.

\section{CMMI SERVICIOS}

El reporte técnico (antecedente \#1) define 24 áreas de proceso que integran buenas prácticas en las cuales se deben centrar los esfuerzos para mejorar los procesos relativos a la prestación de servicios. Cada organización tiene libertad para elegir entre dichas áreas y sobre éstas aplicar la metodología.

Para determinar el nivel en el que se encuentra una compañía es necesario analizar la capacidad y la madurez de las áreas de proceso o de un conjunto de las mismas. A medida que se vaya avanzando en la metodología y se vayan solucionando las no conformidades encontradas, se llevará a cabo una mejora de procesos que supondrá un ascenso en los niveles de capacidad y madurez de CMMI Servicios.

Los niveles de capacidad se aplican al logro de mejora en áreas de proceso individuales. Estos niveles son un medio para mejorar incrementalmente los procesos correspondientes a un área de proceso dada. Los cuatro niveles de capacidad están numerados de 0 a 3.

Los niveles de madurez se aplican al logro de mejora en múltiples áreas de proceso. Estos niveles son un medio para mejorar los procesos correspondientes a un conjunto dado de áreas de proceso (es decir, nivel de madurez). Los cinco niveles de madurez se numeran del 1 al 5. 
Los niveles de capacidad y madurez en los cuales se puede encontrar una organización se muestran a continuación:

\begin{tabular}{|c|ll|}
\hline Nivel & \multicolumn{1}{|c|}{$\begin{array}{c}\text { Representación continua } \\
\text { Niveles de capacidad }\end{array}$} & \multicolumn{1}{c|}{$\begin{array}{c}\text { Representación escalonada } \\
\text { Niveles de madurez }\end{array}$} \\
\hline $\mathbf{0}$ & Incompleto & Inicial \\
$\mathbf{1}$ & Realizado & Administrado \\
$\mathbf{2}$ & Administrado & Definido \\
$\mathbf{3}$ & Definido & Gestionado cuantitativamente \\
$\mathbf{4}$ & & Optimizado \\
$\mathbf{5}$ & & \\
\hline
\end{tabular}

Fuente: Elaboración propia a partir del Reporte Técnico de CMMI Servicios, Versión 1.3.

Adicionalmente, cada área de estudio se puede representar en dos tipos de gráficos: continuo o escalonado.

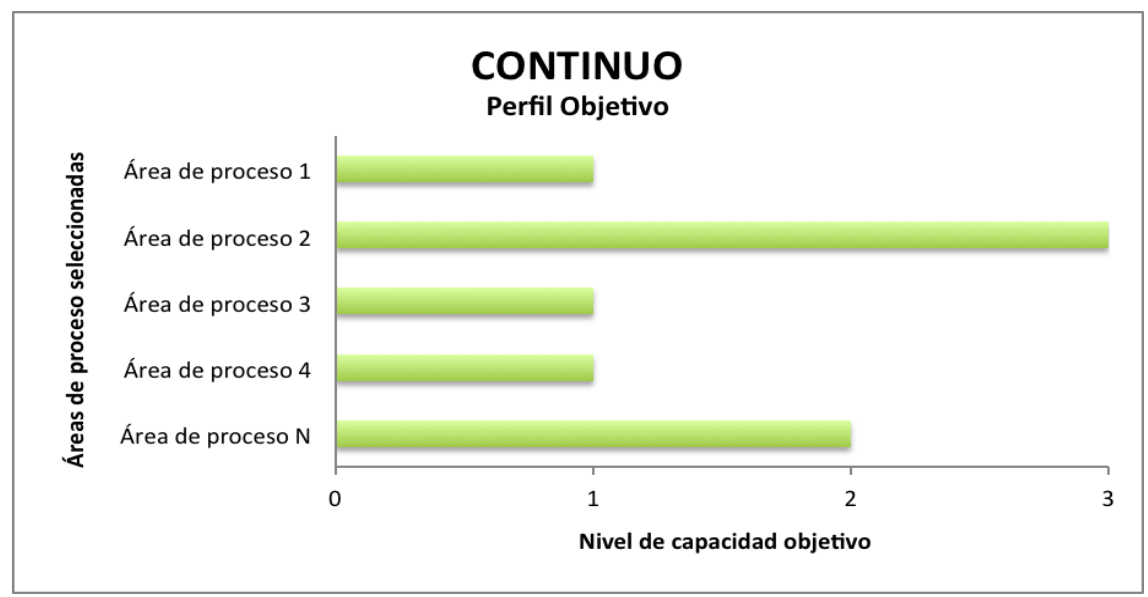

Fuente: Elaboración propia a partir del Reporte Técnico de CMMI Servicios, Versión 1.3.

La representación continua permite enfocar los esfuerzos de mejora de las compañías, eligiendo las áreas que más benefician a la organización y a sus objetivos de negocio. Luego de seleccionar las áreas de proceso, se debe establecer cuánto se desea madurar los procesos asociados a esas áreas, es decir, escoger el nivel de capacidad adecuado para cada una. La selección de una combinación de áreas de proceso y niveles de capacidad normalmente se describe en un "perfil objetivo". Un perfil objetivo define todas las áreas de proceso a tratar y el nivel de capacidad objetivo para cada uno. Este perfil regula las metas y prácticas que la organización abordará en sus esfuerzos de mejora de procesos. 


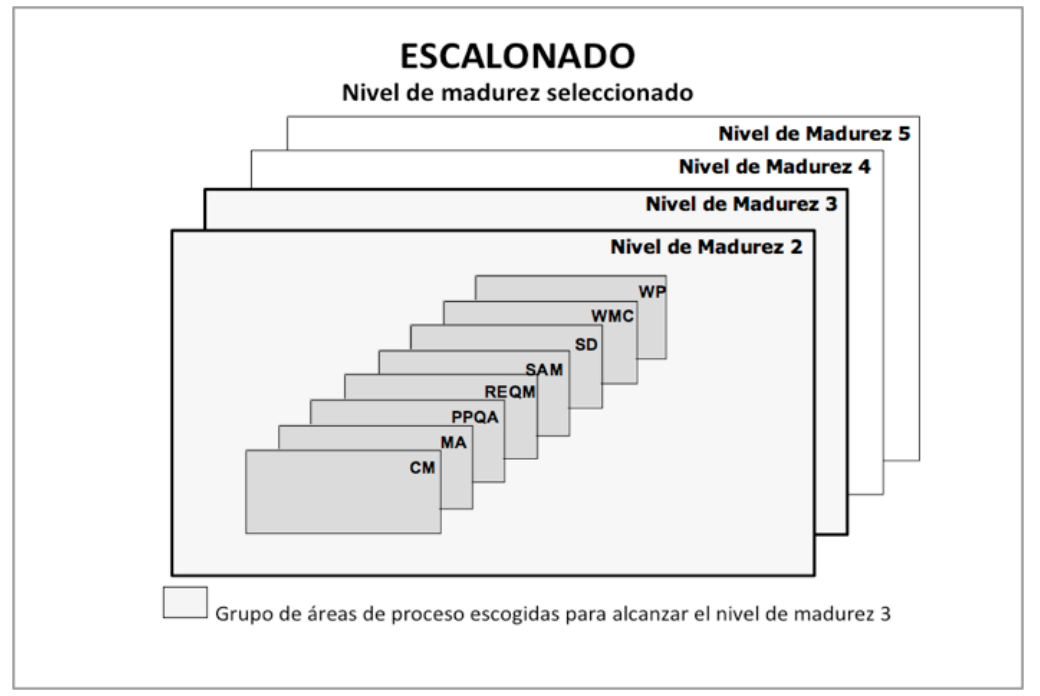

Fuente: Elaboración propia a partir del Reporte Técnico de CMMI Servicios, Versión 1.3.

La representación escalonada proporciona un camino de mejora desde el nivel de madurez 1 hasta el nivel de madurez 5 que implica el logro de los objetivos de las áreas de proceso en cada nivel de madurez. Las áreas de proceso se agrupan por niveles de madurez, indicando qué áreas de proceso implementar para alcanzar cada nivel, es decir, en cada nivel de madurez, hay un conjunto de áreas de proceso que una organización usaría para guiar su mejora de procesos hasta que pueda alcanzar todos los objetivos de todas estas áreas de proceso. Una vez alcanzado dicho nivel de madurez, la organización enfoca sus esfuerzos en las áreas de proceso del siguiente nivel, y así sucesivamente.

\section{METODOLOGÍA PROPUESTA}

Con el fin de brindar a las pequeñas y medianas empresas la posibilidad de aplicación del modelo CMMI-SVC en su negocio, se propone la siguiente metodología:

\section{FASE 0: Requisitos previos para la implantación del modelo}

1. Definición alcance general del proyecto y objetivos de implementación

2. Definición integrantes del equipo y roles

3. Elaboración de análisis de procesos (diagnóstico inicial)

4. Aprobación del plan de acción por parte de la gerencia

5. Formación y capacitación de los integrantes del equipo en el modelo CMMI

6. Publicación del plan de acción

7. Institucionalización del plan de acción

\section{FASE 1: Implantación del modelo en la empresa}

1. Comprensión del área de procesos

2. Definición del plan de trabajo

3. Definición de criterios para determinar la incorporación o modificación de servicios

\section{FASE 2: Análisis del estado de los servicios y levantamiento de necesidades}

1. Identificación del entorno, servicios o buenas prácticas de los mismos

2. Evaluación de los servicios existentes, sus características, acuerdos e interacción con stakeholders 


\section{FASE 3: Creación de propuesta de servicios a implementar}

1. Creación de categorías de los servicios a implementar

2. Creación de acuerdos de niveles de servicio

\section{FASE 4: Aprobación y creación del plan de implementación de servicios}

1. Selección y aprobación de procesos a implementar

2. Creación del plan de implementación

Las fases mencionadas anteriormente fueron planteadas con base en lo propuesto en el antecedente \# 2, complementándolos con apreciaciones propias acerca de las falencias que posee dicha metodología. Se considera importante la aprobación por parte de la gerencia, formación, publicación e institucionalización, con el fin de lograr el compromiso y la implicación de todo el personal en la aplicación del modelo.

Finalmente, de acuerdo con los conceptos del modelo CMMI-SVC y con la metodología propuesta, las pequeñas y medianas empresas podrán utilizar esta herramienta como un elemento clave en la mejora y evaluación de sus procesos.

\section{VENTAJAS DE APLICACIÓN EN PEQUEÑAS Y MEDIANAS EMPRESAS}

Algunas de las ventajas que se pueden obtener con la aplicación del modelo CMMI en PYMES son (Éstas fueron extraídas de la información contenida en el antecedente \# 3, así como percepciones propias acerca de los beneficios de $\mathrm{CMMI}$ ):

- Oportunidad de mejora global en la compañía, evitando centrarse únicamente en partes específicas del negocio, es decir, este modelo está encaminado a abarcar todo el ciclo de vida del producto hasta su entrega y mantenimiento, en lugar de enfocarse en mejoras locales.

- Claridad y transparencia con cada uno de los clientes internos y externos en cuanto a la definición de niveles de acuerdos de servicio.

- Implicación del personal en la mejora y evaluación de procesos.

- Mejora de la relación e interacción entre las diferentes áreas de la compañía.

- Disposición de información de gestión útil a la hora de tomar decisiones ya sean relacionadas con la prestación del servicio o con la mejora continua del proceso.

- Mejora de la satisfacción tanto de clientes internos como externos.

- Estandarización de procesos logrando mejorar la productividad de los mismos.

- Aumento de la competitividad y eficiencia.

- Crecimiento equilibrado y sostenido. 


\section{CONCLUSIONES}

- CMMI-SVC es un modelo de mejora y evaluación de procesos que ayuda no sólo a incrementar su calidad para brindar un buen servicio a los clientes sino que es una herramienta que consigue implicar al personal en la consecución de objetivos, mediante la participación de los trabajadores en la aplicación del modelo.

- Mediante la aplicación del modelo CMMI se les permite a las empresas adquirir un factor de competitividad y eficiencia que será de gran importancia para las PYMES que se encuentran en proceso de expansión tanto nacional como internacionalmente, con el fin de lograr un crecimiento gradual, equilibrado y sostenido, que les permita convertirse en organizaciones exitosas en el medio en el que se desenvuelven.

- Se considera que la metodología propuesta en el presente artículo, para aplicar CMMI Servicios en pequeñas y medianas empresas, es simple y sencilla para que sea fácil de implantar, a un bajo coste y con beneficios visibles para las organizaciones. De esta forma, se logra brindar una herramienta que podría solucionar la falta de aplicación de CMMI para este tipo de compañías, como se expresa en el antecedente \#4. 


\section{REFERENCIAS BIBLIOGRÁFICAS}

[1] Echavarría Ramírez, Andrés Felipe; Nader Ceballos, Jacobo. "Uso del área de proceso Service Delivery (SD) de CMMI For Services, Version 1.3 como guía para servicios en bibliotecas universitarias en el Valle del Cauca" [en línea], Cali: Universidad ICESI, 2012.
[Consulta:
4 mayo
2017].
Disponible
en:

http://repository.icesi.edu.co/biblioteca_digital/bitstream/10906/68356/1/procesos_se rvicios_delivery.pdf

[2] Puello, O.R. "Modelo de verificación y validación basado en CMMI". Investigaciones e innovación en ingenierías [en línea], 2013, vol. 1, n 1, p. 20-27. [Consulta: 4 mayo 2017].
ISSN
2344-8652.
Disponible
en:

http://publicaciones.unisimonbolivar.edu.co/rdigital/ojs/index.php/innovacioning/articl e/view/478

[3] Software Engineering Institute. "CMMI for Services, Version 1.3" [en línea] Pittsburg: Carnegie Mellon University, 2010. [Consulta: 4 mayo 2017]. Disponible en: http://www.sei.cmu.edu/reports/10tr034.pdf

[4] Staples, Mark; et al. "An exploratory study of why organizations do not adopt CMMI" Journal of Systems and Software [en línea], 2007, vol. 80, n 6, p. 883-895. [Consulta: 4 mayo 2017].

Disponible

en:

http://www.sciencedirect.com/science/article/pii/S0164121206002573\# 